\title{
Taking TIMP3 to heart
}

\section{By Lauren Martz, Staff Writer}

A U.S. team has found a way to harness the cardioprotective activity of tissue inhibitor of metalloproteinases 3 while avoiding its off-target effects by embedding it in a hydrogel for direct injection into the heart. ${ }^{1}$ Although preclinical data show that local delivery of the molecule could help prevent heart failure after myocardial infarction in patients with ventricular dilation, it may have a detrimental effect in other patients, making patient selection critical.

After myocardial infarction (MI), left ventricular wall damage is partly caused by overactive matrix metalloproteinase (MMP) enzymes that break down extracellular matrix in the ischemic tissue.

\section{"Using the hydrogel} delivery system was an ingenious way to localize the sustained release of TIMP3 to the site of MI." -Merry Lindsey,

The University of Mississippi Medical Center

South Carolina School of Medicine. The study also included researchers from the University of Pennsylvania and Amgen Inc.

\section{Straight to the heart}

The team encapsulated recombinant TIMP3 (rTIMP3) in a biodegradable hyaluronic acid gel and obtained slow release of rTIMP3 in vitro that maintained a uniform release profile for at least 14 days.

The hydrogel also produced the desired kinetics in vivo. In pigs, rTIMP3 hydrogels remained at the left ventricular injection site for at least seven days after injection. No rTIMP3 was detected in the liver, kidneys, lungs or blood.

In a pig model of MI, rTIMP3 hydrogel injection into the left ventricle decreased left ventricle dilation, wall stress and regional infarct expansion and partially increased left ventricle wall thickness compared with injection of saline or unloaded hydrogels.

rTIMP3 hydrogel-treated pigs had lower pulmonary capillary wedge pressure than saline- or unloaded gel-treated pigs, indicating a decreased risk of progression to heart failure.

The team also showed that rTIMP3 decreased inflammatory markers and increased collagen content in left ventricular myocardial cells compared with saline or unloaded hydrogels.

Because some hydrogels without embedded therapeutics have been used to affect infarct expansion, ${ }^{4}$ the team showed that most of

Under normal conditions, tissue inhibitors of metalloproteinases (TIMPs) control MMP activity and help regulate the breakdown of matrix proteins. After MI, however, there is an increase in MMP plasma levels without a matching change in levels of TIMPs, creating an imbalance in MMP regulation.

The unchecked proteolysis causes thinning of the left ventricle wall, dilation and eventual loss of structural support-termed left ventricular remodeling-which leads to heart failure.

Previous clinical studies have tested various MMP inhibitors. ${ }^{2}$ However, according to Merry Lindsey, problems in achieving proper doses and side effects of systemic administration of MMP inhibitors have prevented clinical use.

Lindsey is director of The University of Mississippi Medical Center's Jackson Center for Heart Research, a professor of physiology and biophysics at the University of Mississippi Medical Center and director of the San Antonio Cardiovascular Proteomics Center at The University of Texas Health Science Center at San Antonio.

Tissue inhibitor of metalloproteinases 3 (TIMP3) was pegged as a key player in the heart in 2009 when knockout studies in mice showed that eliminating Timp 3 causes adverse ventricular remodeling and accelerates heart failure. ${ }^{3}$

To find a therapeutic strategy that can block MMP activity in patients, Francis Spinale and colleagues decided to test whether increasing TIMP3 levels exclusively in the heart via a slow-release delivery system could improve cardiac recovery following MI without incurring effects in other organs.

Spinale is a professor of cell biology and anatomy at the University of the effects were caused by rTIMP3 and not the gel by boiling the protein to destroy its activity. Boiled rTIMP3-loaded hydrogels lacked cardioprotective effects.

Together, the data suggest that TIMP3 acts as a cardioprotective agent after MI by stabilizing the extracellular matrix to improve left ventricular structure and by dampening the MI-associated inflammation.

Data were published in Science Translational Medicine.

LoneStar Heart Inc. has Algisyl-LVR, a hydrogel injected into the left ventricle wall during surgery, in Phase II/III testing for heart failure. The company is also developing a minimally invasive version of the hydrogel for catheter-based delivery, which is in preclinical development. Ventrix Inc. has the injectable hydrogel VentriGel in preclinical testing for congestive heart failure.

\section{Local hero}

According to Lindsey, the localized hydrogel delivery system should minimize the risk of off-target effects.

"Using the hydrogel delivery system was an ingenious way to localize the sustained release of TIMP3 to the site of MI," she said.

Patrick Hsieh, David Lundy and Yi-Dong Lin told SciBX that although many other approaches have focused on delivery of growth factors, extracellular matrix proteins or living cells to the infarct area, "the main advantage of the recombinant TIMP3 approach is the direct targeting of the left ventricular remodeling by MMPs, caused by the MMP/TIMP3 imbalance responsible for progression to heart failure after MI."

However, they added that confining the gel to the damaged area will 
be important because if it spreads to neighboring healthy regions, it could weaken the tissue and cause side effects. Previous attempts to inject substances into the heart have run into problems with insufficient retention within the area or inadequate release beyond the immediate injection site.

Hsieh is a joint associate research fellow at Academia Sinica's Institute of Biomedical Sciences. Lundy and Lin are postdoctoral fellows at Academia Sinica.

Nikolaos Frangogiannis told SciBX that the delivery timing will need to be optimized.

"Infarct healing is a dynamic process. There is a window of therapeutic opportunity - if this is missed, then the result of an intervention may be detrimental."

Frangogiannis is a professor of medicine and chair in cardiovascular medicine at the Albert Einstein College of Medicine of Yeshiva University.

The Academia Sinica team added that although there is extensive remodeling during the first two weeks, those processes continue for at least two months after MI.

Lindsey said that because the results came from injections given at the time of MI, it will be necessary to see the effect of rTIMP3 given after the heart has undergone some irreversible ischemic injury. This would indicate whether TIMP3 can reverse and not just prevent the adverse remodeling.

\section{Patient benefits}

Florence Pinet told SciBX that current treatments-which include acute reperfusion strategies and anti-remodeling medications such as angiotensin-converting enzyme (ACE) inhibitors and $\beta$-blockers-do not prevent ventricle remodeling in about $30 \%$ of patients.

Pinet is research director at the Institut National de la Sante et de la Recherche Medicale (INSERM) UMR744.

However, selecting the right patient population could be difficult.

According to Frangogiannis, clinical heart failure is often associated with ventricular dilation and dysfunction, and patients with large infarcts might benefit from the rTIMP3 hydrogels.

One problem, he said, is that not all heart attack patients develop dilation following infarction, and some patients develop heart failure without dilation. In the latter, heart failure can be caused by overactive matrix deposition leading to formation of a stiff scar and diastolic heart failure.

"The therapy proposed here may be effective for patients with a defect in scar formation that tend to exhibit dilation of the heart following infarction but not for other groups with different physiological responses." He added that patients with overactive fibrotic responses may be negatively affected by the treatment because of the increase in collagen deposition that results.

Imaging or biomarker approaches could help select for patients at high risk of dilative responses, he said.

Another issue, said Frangogiannis, is that most MI patients do not require surgery and are treated with percutaneous interventions or pharmacologic therapies for rapid reperfusion.

"Development of a catheter-based strategy to deliver the hydrogelTIMP3 would be a major step forward," he said.

Frangogiannis added that although the post-MI group would be best suited for the rTIMP3 hydrogels, the gels may also help patients with chronic heart failure who exhibit progressive dilative remodeling.

Spinale told SciBX that an undisclosed entity has filed a patent application covering the use of rTIMP3 hydrogels in arthritis, vascular inflammation, heart failure and related diseases. The IP is unlicensed and currently unavailable for licensing.

Martz, L. SciBX 7(9); doi:10.1038/scibx.2014.246

Published online March 6, 2014

\section{REFERENCES}

1. Eckhouse, S.R. et al. Sci. Transl. Med.; published online Feb. 12, 2014; doi:10.1126/scitranslmed.3007244

Contact: Francis G. Spinale, University of South Carolina School of Medicine, Charleston, S.C.

e-mail: cvctrc@uscmed.sc.edu

2. Dormán, G. et al. Drugs 70, 949-964 (2010)

3. Kassiri, Z. et al. J. Biol. Chem. 284, 29893-29904 (2009)

4. Mukherjee, R. et al. Ann. Thorac. Surg. 86, 1268-1276 (2008)

\section{COMPANIES AND INSTITUTIONS MENTIONED}

Academia Sinica, Taipei, Taiwan

Albert Einstein College of Medicine of Yeshiva University, New York, N.Y.

Amgen Inc. (NASDAQ:AMGN), Thousand Oaks, Calif. Institut National de la Sante et de la Recherche Medicale, Lille, France

LoneStar Heart Inc., Laguna Hills, Calif.

San Antonio Cardiovascular Proteomics Center, San Antonio, Texas

The University of Mississippi Medical Center, Jackson, Miss. University of Pennsylvania, Philadelphia, Pa.

University of South Carolina School of Medicine, Columbia, S.C The University of Texas Health Science Center at San Antonio, San Antonio, Texas

Ventrix Inc., San Diego, Calif. 